\title{
Changes in erythrocyte deformability during day and possible role of melatonin
}

\author{
Rastislav VAzan ${ }^{1}$, Katarina Plauterova ${ }^{1}$, Gabriela Porubska ${ }^{1}$, Jana Radosinska ${ }^{1,2}$ \\ ${ }^{1}$ Institute of Physiology, Faculty of Medicine, Comenius University in Bratislava, Slovak Republic; ${ }^{2}$ Institute for Heart \\ Research, Slovak Academy of Sciences, Bratislava, Slovak Republic \\ E-mail: rastislav.vazan@fmed.uniba.sk
}

Objectives. The deformability of erythrocytes is their ability to change shape in order to pass through the capillaries. This is necessary for quality of microcirculation and sufficient delivery of oxygen to the tissues. The aim of our study was to investigate the possible spontaneous changes in the erythrocyte deformability during day and evaluation of the possible direct effects of melatonin (hormone involved in regulation of biorhythms) on the erythrocyte deformability.

Methods. Samples of capillary blood were taken from 12 healthy volunteers in the morning (8:00) and early in the evening (16:30). Determination of erythrocyte deformability was done based on the measurement of their filtrability. It was measured immediately after the sample collection and 2-hour lasting incubation without or with melatonin $(2000 \mu \mathrm{mol} / \mathrm{L})$.

Results. Erythrocyte deformability was significantly lower in the morning (filtrability index: $0.68 \pm 0.01$ morning vs. $0.71 \pm 0.01$ early evening, $\mathrm{p}<0.05)$. The incubation of blood samples with melatonin did not have impact on deformability.

Conclusions. We suggest the presence of diurnal changes in erythrocyte deformability with worse values in the morning that may contribute to higher risk of ischemic attacks in the morning hours. Direct in vitro effect of melatonin on deformability was not observed, but possible in vivo effects cannot be excluded.

Key words: erythrocyte deformability, filtrability, diurnal changes, melatonin

The deformability of erythrocytes (ERY) is an ability of ERY to change their shape and turn back to the previous form. It is one of the most important determinants of their survival in the circulation, as ERY (approximately $7.2 \mu \mathrm{m}$ in diameter) have to pass through narrow capillaries including as small as 3-4 $\mu \mathrm{m}$ in diameter. This is also crucial for effective microcirculation and sufficient delivery of oxygen to the tissues (Schmid-Schonbein 1976). There is a natural assumption that deformability of ERY may undergo some circadian rhythmic changes likewise many other body functions and systems. This assumption is indirectly supported by two studies performed on rats (Berker et al. 2004; Yerer and
Aydogan 2006), but to the best of our knowledge there is no similar study in humans published and no direct observation of circadian rhythm in ERY deformability was done.

The pineal hormone melatonin plays a crucial role in the regulation of biorhythms in general (Csernus and Mess 2003). Melatonin levels in plasma exert a distinct daily rhythm with high levels during the dark time of light/dark cycle. Light decreases melatonin synthesis and release into the circulation (Herichova 2013). Since ERY are in close contact with all hormones including melatonin, it may be anticipated that melatonin may affect their functions including deformability.

Corresponding author: Rastislav Vazan, MD., PhD., Institute of Physiology, Faculty of Medicine, Comenius University in Bratislava, Sasinkova 2, 81372 Bratislava, Slovak Republic; phone: +421 259357 685; e-mail: rastislav.vazan@fmed.uniba.sk. 
Based on the above-mentioned facts, the aim of our study was to investigate the possible spontaneous changes of ERY deformability during the day and to verify possible direct effects of melatonin on ERY deformability in vitro.

\section{Subjects and Methods}

Study design. All participants included in this study were healthy volunteers of both sexes $(n=12$, age $21-42$ years). We excluded persons with any hematological, endocrine or infectious disease, as well as females in menstrual phase of the cycle. The capillary blood was drawn in the morning at 8:00 and early in the evening at 16:30. The participants were fasting in the morning; they had light lunch (before 13:00) and no further food intake untill the second blood collection. The participants were asked to sleep at least 7 hours in dark room during the night before the experiment and avoid any intensive physical activity or sleep during the experimental day. All the experiments were performed from September till January. Design of the experiments conforms to ethical guidelines of the Declaration of Helsinki (The

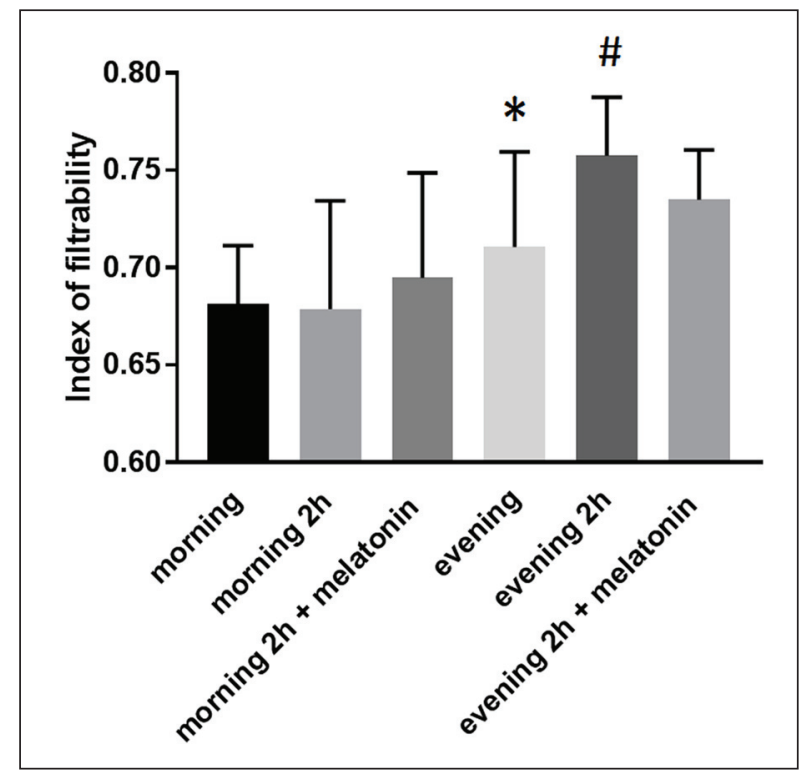

Figure 1. Filtrability of erythrocytes. Explanations: morning - immediate analysis in the morning; morning $2 \mathrm{~h}$ - analysis after 2 hours of incubation without melatonin in the morning; morning $2 \mathrm{~h}+$ melatonin - analysis after 2 hours of incubation with melatonin in the morning; evening - immediate analysis in the evening; evening $2 \mathrm{~h}$ - analysis after 2 hours of incubation without melatonin in the evening, evening $2 \mathrm{~h}+$ melatonin - analysis after 2 hours of incubation with melatonin in the evening. Data are presented as means \pm SD. Statistical significance: ${ }^{*} \mathrm{p}<0.05$ vs. morning, $\# \mathrm{p}<0.05$ vs. morning $2 \mathrm{~h}$.
Code of Ethics of the World Medical Association). Subjects were healthy volunteers informed about all aspects of the experimental protocol. Experiments were undertaken with the understanding and consent of each subject.

Deformability of erythrocytes. The erythrocyte deformability was determined by a filtration method as described previously (Radosinska et al. 2016). Each blood sample was diluted in Cellpack solution (diluent for Sysmex blood analyzer, Japan; 1:500, v:v) immediately after collection. This suspension was divided into 3 experimental samples: sample for immediate analysis, sample for analysis after 2-hour lasting incubation with melatonin in concentration $2000 \mu \mathrm{mol} / \mathrm{L}$, and sample for analysis after 2-hour lasting incubation without melatonin that served as control for melatonin-incubated samples. Melatonin (Sigma-Aldrich, USA) was directly dissolved in Cellpack solution of the corresponding samples. The incubation was performed in dark box at room temperature $\left(21-24^{\circ} \mathrm{C}\right)$. The deformability of ERY was determined according to their filtrability through special membrane filter units (Ultrafree-MC SV Centrifugal Filter, Millipore, Germany) with $5 \mu \mathrm{m}$ pores in diameter. Driving force for filtration was centrifugation at $1400 \mathrm{rpm}$ (Hettich MIKRO 120 centrifuge) for 5 minutes. The count of ERY before and after centrifugation was determined by hematological analyzer Sysmex (Japan) and subsequently the index of filtrability (IF) was calculated. IF represents ratio of filtered ERY to the number of ERY counted before centrifugation. An increase in IF indicates an increase in ERY deformability.

Statistical analysis. The data are presented as means \pm standard deviations (SD). Normality of the data distribution was analyzed according to Kolmogorov-Smirnov. Statistical significance of differences was analyzed by paired t-test for normally distributed values and by Wilcoxon matched pairs test for not normally distributed values. Differences were considered as significant at $\mathrm{p}<0.05$. GraphPad Instat software was used for all the statistical analyses.

\section{Results}

The IF was significantly lower in the morning samples compared with evening samples. This difference was also preserved after 2-hour lasting incubation of samples without melatonin. On the other hand, in the morning as well as in the evening samples, there was no significant difference in IF between the incubated blood samples with or without the presence of melatonin. The results are shown in the Figure 1. 


\section{Discussion}

Humans display changes in gene activity, biochemistry, physiology, and behavior that wax and wane through the cycle of days and nights. The most important result of this study concerns the changes in the deformability of ERY during day, with lower values in the morning and higher values in the early evening. Lower values in the morning (after the dark phase of the day - after natural in vivo exposure of ERY to melatonin in blood) are consistent with result of the animal study of Yerer and Aydogan (2006), where light/dark regimen with 24 hours of darkness caused decrease in deformability. Several studies have demonstrated a circadian pattern to the occurrence of sudden cardiac death and strokes with a higher frequency in the morning hours (Muller et al. 1987; Raj et al. 2015). It is possible that there is some relationship between deformability of ERY, incidence of cardiovascular events, and melatonin (hormone involved in the regulation of biorhythms, produced in the pineal gland during darkness). However, information on relationship between melatonin and deformability of ERY is inconsistent in the literature. It has been shown that administration of melatonin after pinealectomy reduced ERY deformability under in vivo conditions (Berker et al. 2004). In vitro study of Dikmenoglu et al. (2008) has shown melatonin-induced deterioration of ERY deformability under oxidative stress, but on the other side, melatonin alone did not cause any effect on ERY deformability. Melatonin treatment also did not affect decreased erythrocyte deformability and increased erythrocyte aggregation in streptozotocininduced diabetic rats (Gumustekin et al. 2007). These inconsistent findings in the literature may result from differences in experimental conditions or dosage and duration of melatonin treatment, as well as from different methods used for determination of deformability. Nevertheless, changes in ERY deformability were not induced by in vitro incubation of blood with melatonin in our study and mechanism that is responsible for the observed changes of ERY deformability during day needs to be elucidated. There are several possible reasons for no in vitro effect of melatonin on ERY deformability in our experiment. It is possible that only 2-hour incubation or used concentration of melatonin were not sufficient for ERY deformability change. Moreover, in vitro incubation could not reveal possible indirect mechanisms that may be involved in in vivo conditions. Probability of a specific receptor-mediated direct effect of melatonin on ERY is very low, since no melatonin receptors in ERY have been described in the literature up to this time. It is also possible that melatonin has no effect on ERY deformability in general, as these cells have many specifics comparing with other cells (e.g. no nucleus, specific cytoskeleton, and specific more or less anaerobic metabolism). On the other hand, it is possible that longer contact of ERY with melatonin in vivo during night (though in low physiological concentrations) may influence red blood cells via various non-specific direct or indirect mechanisms that cannot be involved in the case of in vitro incubation. Except the direct effect of melatonin on cytoskeleton of the cells (Benitez-King 2006), we can mention one more factor possibly responsible for spontaneous changes of ERY deformability. It is $\mathrm{Na}, \mathrm{K}-\mathrm{ATP}$ ase, enzyme regulating cell volume and consequently ERY deformability (Radosinska and Vrbjar 2016). It has been shown that presence of some blood plasma inhibitor (not identified yet) is responsible for circadian changes in rat renal $\mathrm{Na}, \mathrm{K}$-ATPase activity (Segura et al. 2004) and circadian changes has been also observed on ERY membrane-associated $\mathrm{Na}$,K-ATPase (Chakravarty and Rizvi 2011). It is possible that mentioned plasma inhibitor may influence also this type of Na,K-ATPase and leads to changes of ERY deformability during the course of the day. Since the influence of melatonin on the mentioned plasma inhibitor is not excluded, melatonin can be involved in the changes of ERY deformability indirectly.

We are aware of limitations of our study. Only small group of participants was included, only one concentration of melatonin was applied and duration of the sample incubation was methodologically limited. In spite of this, we hope that our original human study that is in accord with results of animal studies of other authors could help to other researchers in scheduling their experiments in view of the fact that erythrocyte deformability is not stable during the course of day.

\section{Acknowledgements}

This research was supported by grant VEGA SR 1/0032/14. The authors thank to Dr. Anna Mataseje for professional support and Jana Meryova and Dagmar Ciganekova for their excellent technical assistance.

\section{References}

Berker M, Dikmenoglu N, Bozkurt G, Ergonul Z, Ozgen T. Hemorheology, melatonin and pinealectomy. What's the relationship? An experimental study. Clin Hemorheol Microcirc 30, 47-52, 2004. 
Benitez-King G. Melatonin as a cytoskeletal modulator: implications for cell physiology and disease. J Pineal Res 40, $1-9,2006$.

Chakravarty S, Rizvi SI. Circadian modulation of sodium-potassium ATPase and sodium - proton exchanger in human erythrocytes: in vitro effect of melatonin. Cell Mol Bio 57, 80-86, 2011.

Csernus V, Mess B. Biorhythms and pineal gland. Neuro Endocrinol Lett 24, 404-411, 2003.

Dikmenoglu N, Ileri E, Seringec N, Ercil D. Melatonin prevents lipid peroxidation in human erythrocytes but augments deterioration of deformability after in vitro oxidative stress. Clin Hemorheol Microcirc 40, 235-242, 2008.

Gumustekin M, Tekmen I, Guneli E, Tugyan K, Topcu A, Ergonen AT, Ozdemir MH, Uysal N, Bediz CS. Short-term melatonin treatment improved diabetic nephropathy but did not affect hemorheological changes in diabetic rats. Pharmazie 62, 693-698, 2007.

Herichova I. Changes of physiological functions induced by shift work. Endocr Regul 47, 159-170, 2013.

Muller JE, Ludmer PL, Willich SN, Tofler GH, Aylmer G, Klangos I, Stone PH. Circadian variation in the frequency of sudden cardiac death. Circulation 75, 131-138, 1987.

Radosinska J, Vrbjar N. The role of red blood cell deformability and Na,K-ATPase function in selected risk factors of cardiovascular diseases in humans: focus on hypertension, diabetes mellitus and hypercholesterolemia. Physiol Res 65 Suppl 1, S43-S54, 2016.

Radosinska J, Mezesova L, Okruhlicova L, Frimmel K, Breierova E, Bartekova M, Vrbjar N. Effect of yeast biomass with high content of carotenoids on erythrocyte deformability, NO production and Na,K-ATPase activity in healthy and LPS treated rats. Clin Hemorheol Microcirc 64, 125-134, 2016.

Raj K, Bhatia R, Prasad K, Srivastava MV, Vishnubhatla S, Singh MB. Seasonal differences and circadian variation in stroke occurrence and stroke subtypes. J Stroke Cerebrovasc Dis 24, 10-16, 2015.

Schmid-Schonbein H. Microrheology of erythrocytes, blood viscosity, and the distribution of blood flow in the microcirculation. Int Rev Physiol 9, 1-62, 1976.

Segura D, Eblen-Zajjur A, Proverbio F, Proverbio T, Carrera F, Caruso-Neves C, Marin R. A blood plasma inhibitor is responsible for circadian changes in rat renal Na,K-ATPase activity. Int J Biochem Cell Biol 36, 2054-2065, 2004.

Yerer MB, Aydogan S. The importance of circadian rhythm alterations in erythrocyte deformability. Clin Hemorheol Microcirc 35, 143-147, 2006. 